\title{
Bipolar Fuzzy Trees
}

\author{
Muhammad Akram ${ }^{1}$ and Adeel Farooq ${ }^{2}$ \\ ${ }^{1}$ Punjab University College of Information Technology,University of the Punjab, Old Campus,Lahore-54000, Pakistan \\ ${ }^{2}$ Department of Mathematics, COMSATS Institute of Information Technology, Lahore, Pakistan
}

Received: 2 March 2016, Accepted: 17 March 2016

Published online: 19 June 2016.

\begin{abstract}
Connectivity has an important role in different disciplines of computer science including computer network. In the design of a network, it is important to analyze connections by the levels. The structural properties of bipolar fuzzy graphs provide a tool that allows for the solution of operations research problems. In this paper, we introduce various types of bipolar fuzzy bridges, bipolar fuzzy cut-vertices, bipolar fuzzy cycles and bipolar fuzzy trees in bipolar fuzzy graphs, and investigate some of their properties. Most of these various types are defined in terms of levels. We also describe comparison of these types.
\end{abstract}

Keywords: Bipolar fuzzy cycles, bipolar fuzzy trees, bipolar fuzzy bridges, and bipolar fuzzy cut-vertices, levels.

\section{Introduction}

Graph theory has numerous applications to problems in computer science, electrical engineering, system analysis, operations research, economics, networking routing, and transportation. However, in many cases, some aspects of a graph-theoretic problem may be uncertain. For example, the vehicle travel time or vehicle capacity on a road network may not be known exactly. In such cases, it is natural to deal with the uncertainty using the methods of fuzzy sets and fuzzy logic. A (crisp) set $A$ in a universe $X$ can be defined in the form of its characteristic function $\mu_{A}: X \rightarrow\{0,1\}$ yielding the value 1 for elements belonging to the set $A$ and the value 0 for elements excluded from the set $A$. The most of the generalization of the crisp set have been introduced on the unit interval $[0,1]$ and they are consistent with the asymmetry observation. In other words, the generalization of the crisp set to fuzzy sets [19] relied on spreading positive information that fit the crisp point $\{1\}$ into the interval $[0,1]$. The theory of fuzzy sets has become a vigorous area of research in different disciplines including medical and life sciences, management sciences, social sciences, engineering, statistics, graph theory, artificial intelligence, signal processing, multiagent systems, pattern recognition, robotics, computer networks, expert systems, decision making and automata theory. There have been several generalizations of this fundamental concept. In 1994, Zhang [22] initiated the concept of bipolar fuzzy sets as a generalization of fuzzy sets [19]. Bipolar fuzzy sets are an extension of fuzzy sets whose membership degree range is $[-1,1]$. In a bipolar fuzzy set, the membership degree 0 of an element means that the element is irrelevant to the corresponding property, the membership degree $(0,1]$ of an element indicates that the element somewhat satisfies the property, and the membership degree $[-1,0)$ of an element indicates that the element somewhat satisfies the implicit counter-property.

Kaufmann's initial definition of a fuzzy graph [9] was based on Zadeh's fuzzy relations [20]. Rosenfeld [17] introduced the fuzzy analogue of several basic graph-theoretic concepts including bridges, cut-nodes, connectedness, trees and cycles. Bhattacharya [7] gave some remarks on fuzzy graphs, and Sunitha and Vijayakumar [18] characterized fuzzy trees. Bhutani and Rosenfeld [8] introduced the concepts of strong arcs, fuzzy end nodes and geodesics in fuzzy graphs 
and types of arcs in a fuzzy graph are described in [11]. Akram et al.[1-5] has introduced many concepts, including bipolar fuzzy graphs, regular bipolar fuzzy graphs, bipolar fuzzy hypergraphs and metric aspects of bipolar fuzzy graphs. In this paper, we introduce various types of bipolar fuzzy bridges, bipolar fuzzy cut-vertices, bipolar fuzzy cycles and bipolar fuzzy trees in bipolar fuzzy graphs, and investigate some of their interesting properties. Most of these various types are defined in terms of levels. We also describe comparison of these types.

We have used standard definitions and terminologies in this paper. For other notations, terminologies and applications not mentioned in the paper, the readers are referred to [10-13 19, 20].

\section{Preliminaries}

In this section, we review some elementary concepts whose understanding is necessary fully benefit from this paper.

By a graph, we mean a pair $G^{*}=(V, E)$, where $V$ is the set and $E$ is a relation on $V$. The elements of $V$ are vertices of $G^{*}$ and the elements of $E$ are edges of $G^{*}$. We write $x y \in E$ to mean $(x, y) \in E$, and if $e=x y \in E$, we say $x$ and $y$ are adjacent. A path in a graph $G^{*}$ is an alternating sequence of vertices and edges $v_{0}, e_{1}, v_{1}, e_{2}, \cdots, v_{n-1}, e_{n}, v_{n}$. The path graph with $n$ vertices is denoted by $P_{n}$. A path is sometime denoted by $P_{n}: v_{0} v_{1} \cdots v_{n}(n>0)$. The length of a path $P_{n}$ in $G^{*}$ is $n$. A path $P_{n}: v_{0} v_{1} \cdots v_{n}$ in $G^{*}$ is called a cycle if $v_{0}=v_{n}$ and $n \geq 3$. Note that path graph, $P_{n}$, has $n-1$ edges and can be obtained from cycle graph, $C_{n}$, by removing any edge. An undirected graph $G^{*}$ is connected if there is a path between each pair of distinct vertices. A block is a maximal biconnected subgraph of a given graph $G$. An edge $e$ in a connected graph $G$ is a bridge (cut-edge or cut arc) if $G-e$ is disconnected. A vertex $v$ in a connected graph $G$ is a cut-vertex if $G-v$ is disconnected. The graphs with exactly $n-1$ bridges are exactly the trees, and the graphs in which every edge is a bridge are exactly the forests. A spanning tree in a connected graph $G$ is a subgraph of $G$ that includes all the vertices of $G$ and is also a tree. A forest is an undirected graph, all of whose connected components are trees; in other words, the graph consists of a disjoint union of trees.

A fuzzy subset $\mu$ on a set $X$ is a map $\mu: X \rightarrow[0,1]$. A fuzzy binary relation $v$ on $X$ is a fuzzy subset $v$ on $X \times X$. By a fuzzy relation $v$, we mean a fuzzy binary relation given by $v: X \times X \rightarrow[0,1]$. Let $v \circ v$ be a fuzzy set of $E \subseteq V \times V$ defined by $v \circ v(x, y)=\sup \{\min \{v(x, y), v(y, z)\} \mid z \in V\}$. Then $v \circ v$ is called the composition of $v$ with itself. Since composition is associative, we get $v^{k}=v^{k-1} \circ v$ for $k=1,2,3, \cdots$. Define the fuzzy subset $v^{\infty}$ of $V \times V$ by

$$
v^{\infty}(x, y)=\sup \left\{v^{k}(x, y): k=1,2, \cdots\right\}
$$

$v^{\infty}(x, y)$ denotes the "strength of connectedness" between two nodes $x$ and $y$. That is, $v^{\infty}(x, y)$ is defined as the maximum of the strengths of all paths between $x$ and $y$.

Definition 1. [12,22] Let $X$ be a nonempty set. A bipolar fuzzy set $B$ in $X$ is an object having the form

$$
B=\left\{\left(x, \mu_{B}^{P}(x), \mu_{B}^{N}(x)\right) \mid x \in X\right\}
$$

where $\mu_{B}^{P}: X \rightarrow[0,1]$ and $\mu_{B}^{N}: X \rightarrow[-1,0]$ are mappings.

We use the positive membership degree $\mu_{B}^{P}(x)$ to denote the satisfaction degree of an element $x$ to the property corresponding to a bipolar fuzzy set $B$, and the negative membership degree $\mu_{B}^{N}(x)$ to denote the satisfaction degree of an element $x$ to some implicit counter-property corresponding to a bipolar fuzzy set $B$. If $\mu_{B}^{P}(x) \neq 0$ and $\mu_{B}^{N}(x)=0$, it is the situation that $x$ is regarded as having only positive satisfaction for $B$. If $\mu_{B}^{P}(x)=0$ and $\mu_{B}^{N}(x) \neq 0$, it is the situation that $x$ 
does not satisfy the property of $B$ but somewhat satisfies the counter property of $B$. It is possible for an element $x$ to be such that $\mu_{B}^{P}(x) \neq 0$ and $\mu_{B}^{N}(x) \neq 0$ when the membership function of the property overlaps that of its counter property over some portion of $X$.

For the sake of simplicity, we shall use the symbol $B=\left(\mu_{B}^{P}, \mu_{B}^{N}\right)$ for the bipolar fuzzy set

$$
B=\left\{\left(x, \mu_{B}^{P}(x), \mu_{B}^{N}(x)\right) \mid x \in X\right\} .
$$

Definition 2.[22] Let $X$ be a nonempty set. Then we call a mapping $A=\left(\mu_{A}^{P}, \mu_{A}^{N}\right): X \times X \rightarrow[0,1] \times[-1,0]$ a bipolar fuzzy relation on $X$ such that $\mu_{A}^{P}(x, y) \in[0,1]$ and $\mu_{A}^{N}(x, y) \in[-1,0]$.

Definition 3.[12] The support of a bipolar fuzzy set $A=\left(\mu_{A}^{P}, \mu_{A}^{N}\right)$, denoted by $\operatorname{supp}(A)$, is defined by

$$
\operatorname{supp}(A)=\operatorname{supp}^{P}(A) \cup \operatorname{supp}^{N}(A), \operatorname{supp}^{P}(A)=\left\{x \mid \mu_{A}^{P}(x)>0\right\}, \operatorname{supp}^{N}(A)=\left\{x \mid \mu_{A}^{N}(x)<0\right\} .
$$

We call $\operatorname{supp}^{P}(A)$ as positive support and $\operatorname{supp}^{N}(A)$ as negative support.

Definition 4.[12] Let $A=\left(\mu_{A}^{P}, \mu_{A}^{N}\right)$ be a bipolar fuzzy set on $X$ and let $\alpha \in[0,1]$. $\alpha$-cut $A_{\alpha}$ of $A$ can be defined as

$$
A_{\alpha}=A_{\alpha}^{P} \cup A_{\alpha}^{N}, A_{\alpha}^{P}=\left\{x \mid \mu_{\alpha}^{P}(x) \geq \alpha\right\}, A_{\alpha}^{P}=\left\{x \mid \mu_{\alpha}^{N}(x) \leq-\alpha\right\} .
$$

We call $A_{\alpha}^{P}$ as positive $\alpha$-cut and $A_{\alpha}^{N}$ as negative $\alpha$-cut. The height of a bipolar fuzzy set $A=\left(\mu_{A}^{P}, \mu_{A}^{N}\right)$ is defined as $h(A)=\max \left\{\mu_{A}^{P}(x) \mid x \in X\right\}$. The depth of a bipolar fuzzy set $A=\left(\mu_{A}^{P}, \mu_{A}^{N}\right)$ is defined as $d(A)=\min \left\{\mu_{A}^{N}(x) \mid x \in X\right\}$. We shall say that bipolar fuzzy set $A$ is normal, if there is at least one $x \in X$ such that $\mu_{A}^{P}(x)=1$ or $\mu_{A}^{N}(x)=-1$.

Definition 5.[1] A bipolar fuzzy graph $G=(V, A, B)$ is a non-empty set $V$ together with a pair of functions $A=\left(\mu_{A}^{P}, \mu_{A}^{N}\right)$ : $V \rightarrow[0,1] \times[-1,0]$ and $B=\left(\mu_{B}^{P}, \mu_{B}^{N}\right): V \times V \rightarrow[0,1] \times[-1,0]$ such that for all $x, y \in V$,

$$
\mu_{B}^{P}(x, y) \leq \min \left(\mu_{A}^{P}(x), \mu_{A}^{P}(y)\right) \text { and } \mu_{B}^{N}(x, y) \geq \max \left(\mu_{A}^{N}(x), \mu_{A}^{N}(y)\right) .
$$

Notice that $\mu_{B}^{P}(x, y)>0, \mu_{B}^{N}(x, y)<0$ for $(x, y) \in V \times V, \mu_{B}^{P}(x, y)=\mu_{B}^{N}(x, y)=0$ for $(x, y) \notin V \times V$, and $B$ is symmetric relation.

Definition 6.[1] A bipolar fuzzy graph is called complete if $\mu_{B}^{P}(x, y)=\min \left(\mu_{A}^{P}(x), \mu_{A}^{P}(y)\right)$, $\mu_{B}^{N}(x, y)=\max \left(\mu_{A}^{N}(x), \mu_{A}^{N}(y)\right)$ for all $x, y \in V$.

Definition 7. The support of $A$, denoted by $A^{*}$, is defined by

$$
A^{*}=\left(\left(\mu_{A}^{P}\right)^{*},\left(\mu_{A}^{N}\right)^{*}\right)=\left\{x \in V \mid \mu_{A}^{P}(x)>0 \text { and } \mu_{A}^{N}(x)<0\right\} .
$$

The support of $B$, denoted by $B^{*}$, is defined by

$$
B^{*}=\left(\left(\mu_{B}^{P}\right)^{*},\left(\mu_{B}^{N}\right)^{*}\right)=\left\{(x, y) \in E \mid \mu_{B}^{P}(x, y)>0 \text { and } \mu_{B}^{N}(x, y)<0\right\}
$$

Let $G^{*}=\left(A^{*}, B^{*}\right)$. For $s \in(0,1], t \in[-1,0), A^{(s, t)}=\left\{x \in V \mid \mu_{A}^{P}(x) \geq s, \mu_{A}^{N}(x) \leq t\right\}$ is called an $(s, t)$-level subset of $A$, $B^{(s, t)}=\left\{(x, y) \in E \mid \mu_{B}^{P}(x, y) \geq s, \mu_{B}^{N}(x, y) \leq t\right\}$ is called an $(s, t)$-level subset of $B$. Let $G^{(s, t)}=\left(A^{(s, t)}, B^{(s, t)}\right)$.

Definition 8. [4] A path $P$ in a bipolar fuzzy graph $G$ is a sequence of distinct vertices $v_{1}, v_{2}, \cdots, v_{n}$ such that either one of the following condition is satisfied: 
(1) $\mu_{B}^{P}(x, y)>0$ and $\mu_{B}^{N}(x, y)<0$ for some $x, y$.

(2) $\mu_{B}^{P}(x, y)>0$ and $\mu_{B}^{N}(x, y)=0$ for some $x, y$.

(3) $\mu_{B}^{P}(x, y)=0$ and $\mu_{B}^{N}(x, y)<0$ for some $x, y$.

When $\mu_{B}^{P}(x, y)=\mu_{B}^{N}(x, y)=0$ for some $x, y$, then there is no edge between $x$ and $y$. Otherwise, there exists an edge between $x$ and $y$.

Definition 9.[4] A bipolar fuzzy graph $G$ is connected if any two vertices are joined by a path.

Definition 10.[4] If $x, y \in V$, the $\mu^{P}$ - strength of connectedness between $x$ and $y$ is

$$
\begin{gathered}
\left(\mu_{B}^{P}\right)^{\infty}(x, y)=\sup \left\{\left(\mu_{B}^{P}\right)^{k}(x, y) \mid k=1,2, \cdots, n\right\}, \\
\left(\mu_{B}^{P}\right)^{\infty}(x, y)=\sup \left\{\mu_{B}^{P}\left(x, v_{1}\right) \wedge \mu_{B}^{P}\left(v_{1}, v_{2}\right) \wedge \ldots \wedge \mu_{B}^{P}\left(v_{k-1}, y\right) \mid x, v_{1}, v_{2}, \cdots, v_{k-1}, y \in V, k=1,2, \cdots, n\right\} .
\end{gathered}
$$

The $\mu^{N}$ - strength of connectedness between $x$ and $y$ is

$$
\begin{gathered}
\left(\mu_{B}^{N}\right)^{\infty}(x, y)=\inf \left\{\left(\mu_{B}^{N}\right)^{k}(x, y) \mid k=1,2, \cdots, n\right\}, \\
\left(\mu_{B}^{N}\right)^{\infty}(x, y)=\inf \left\{\mu_{B}^{N}\left(x, v_{1}\right) \vee \mu_{B}^{N}\left(v_{1}, v_{2}\right) \vee \ldots \vee \mu_{B}^{N}\left(v_{k-1}, y\right) \mid x, v_{1}, v_{2}, \cdots, v_{k-1}, y \in V, k=1,2, \cdots, n\right\} .
\end{gathered}
$$

The $\mu^{P}$-strength and $\mu^{N}$-strength of connectedness between $x$ and $y$ in $G$ is denoted by $\left(\mu_{G}^{P}\right)^{\infty}(x, y)$ and $\left(\mu_{G}^{N}\right)^{\infty}(x, y)$, respectively. Also $\left(\mu_{B}^{P}\right)^{\prime \infty}(x, y)$ and $\left(\mu_{B}^{N}\right)^{\prime}(x, y)$ denote $\left(\mu_{G-(x, y)}^{P}\right)^{\infty}(x, y)$ and $\left(\mu_{G-(x, y)}^{N}\right)^{\infty}(x, y)$, where $G-(x, y)$ is obtained from $G$ by deleting the arc $(x, y)$.

\section{Bridges, cut-vertices and blocks}

We define here a bipolar fuzzy edge graph $G=(V, B)$ when $V$ is a crisp vertex set and $B=\left(\mu_{B}^{P}, \mu_{B}^{N}\right)$ is a bipolar fuzzy relation on $V$.

Definition 11. A bipolar fuzzy edge graph on a crisp graph $G^{*}=(V, E)$ is an ordered pair of the form $\widehat{G}=(V, B)$, where $V$ is the crisp vertex set, the functions $\mu_{B}^{P}: V \times V \rightarrow[0,1]$ and $\mu_{B}^{N}: V \times V \rightarrow[0,1]$ are defined by $\mu_{B}^{P}(x, y) \leq$ $\min \left\{\mu_{A}(x), \mu_{A}(y)\right\}$ and $\mu_{B}^{N}(x, y) \geq \max \left\{\mu_{A}^{N}(x), \mu_{A}^{N}(y)\right\}$ for all $x y \in$ E. Notice that $\mu_{B}^{P}(x, y)>0, \mu_{B}^{N}(x, y)<0$ for $(x, y) \in$ $V \times V, \mu_{B}^{P}(x, y)=\mu_{B}^{N}(x, y)=0$ for $(x, y) \notin V \times V$, and $B$ is symmetric relation.

Definition 12.[?] A bridge $(x, y)$ in bipolar fuzzy graph $G$ is said to be $\mu^{P}$-bridge, if deleting $(x, y)$ reduces the $\mu^{P}$-strength of connectedness between some pair of vertices. A bridge $(x, y)$ is said to be $\mu^{N}$-bridge, if deleting $(x, y)$ increases the $\mu^{N}$-strength of connectedness between some pair of vertices. A bridge $(x, y)$ is said to be a bipolar fuzzy bridge, if it is $\mu^{P}$-bridge and $\mu^{N}$-bridge.

Definition 13. Let $(x, y) \in E$.

(1) $(x, y)$ is called a bridge if $(x, y)$ is a bridge of $G^{*}=\left(A^{*}, B^{*}\right)$.

(2) $(x, y)$ is called a bipolar fuzzy bridge if $\left(\mu^{\prime}{ }_{B}\right)^{\infty}(u, v)<\left(\mu_{B}^{P}\right)^{\infty}(u, v)$ and $\left(\mu^{N}{ }_{B}\right)^{\infty}(u, v)>\left(\mu_{B}^{N}\right)^{\infty}(u, v)$ for some $(u, v) \in$ $E$, where $\mu^{P}{ }_{B}$ and $\mu^{N}{ }_{B}$ are $\mu_{B}^{P}$ and $\mu_{B}^{N}$ restricted to $V \times V-\{(x, y),(y, x)\}$.

(3) $(x, y)$ is called a weak bipolar fuzzy bridge if there exists $(s, t) \in(0, h(B)] \times[d(B), 0)$ such that (x,y) is a bridge of $G^{(s, t)}$.

(4) $(x, y)$ is called a partial bipolar fuzzy bridge if $(x, y)$ is a bridge for $G^{(s, t)}$ for all $(s, t) \in(d(B), h(B)] \cup\{h(B)\}$.

(5) $(x, y)$ is called a full bipolar fuzzy bridge if $(x, y)$ is a bridge for $G^{(s, t)}$ for all $(s, t) \in(0, h(B)] \times[d(B), 0)$. 
Example 1. Consider a connected bipolar fuzzy graph as shown in the Fig. 1.

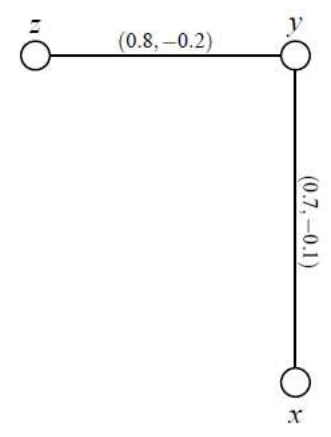

Fig. 1: Connected bipolar fuzzy graph.

By routine computations, we have $d(B)=-0.2, h(B)=0.8$. Thus $(s, t) \in(0,0.8] \times[-0.2,0)$. For $0<s \leq 0.7,-0.2 \leq$ $t<0, G^{(s, t)}=(V,\{(x, y),(y, z)\})$. For $0.7<s \leq 0.8,-0.2 \leq t<0, G^{(s, t)}=(V,\{(y, z)\})$. Hence we conclude that $(y, z)$ is a full bipolar fuzzy bridge and $(x, y)$ is a weak bipolar fuzzy bridge, but not a partial bipolar fuzzy bridge. Both $(x, y)$ and $(y, z)$ are bridges and bipolar fuzzy bridges.

Example 2. Consider a connected bipolar fuzzy graph as shown in the Fig. 2.

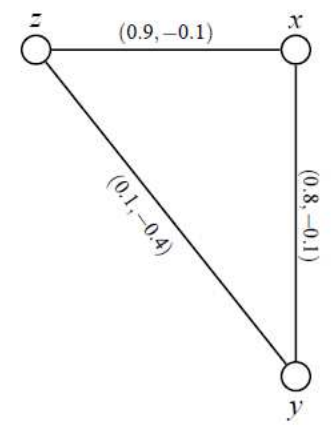

Fig. 2: Connected bipolar fuzzy graph.

By routine computations, we have $d(B)=-0.4, h(B)=0.9$. For $0<s \leq 0.1,-0.4 \leq t<0$, $G^{(s, t)}=(V,\{(x, y),(x, z),(y, z)\})$. For $0.1<s \leq 0.8, \quad-0.1 \leq t<0, \quad G^{(s, t)}=(V,\{(x, y),(x, z)\})$. For $0.8<s \leq 0.9,-0.1 \leq t<0, G^{(s, t)}=(V,\{(x, z)\})$. Thus $(x, z)$ is a bipolar fuzzy bridge and a partial bipolar fuzzy bridge, but not a bridge. The edge $(y, z)$ is not any of five types of bridges.

Example 3. Consider a connected graph $G^{*}=(V, E)$ such that $V=\{x, y, z\}, E=\{(x, y),(y, z),(x, z)\}$. Let $A$ be a bipolar fuzzy set of $V$ and let $B$ be a bipolar fuzzy set of $E \subseteq V \times V$ defined by

$$
\mu_{A}^{P}(x)=\mu_{A}^{P}(y)=\mu_{A}^{P}(z)=1, \mu_{A}^{N}(x)=\mu_{A}^{N}(y)=\mu_{A}^{N}(z)=-1,
$$




$$
\mu_{B}^{P}(x, y)=\mu_{B}^{P}(y, z)=\mu_{B}^{P}(x, z)=0.9, \mu_{B}^{N}(x, y)=\mu_{B}^{N}(y, z)=\mu_{B}^{N}(x, z)=-0.1 .
$$

Routine computations show that connected bipolar fuzzy graph $G$ has no bridges of any of the five types.

Example 4. Consider a connected graph $G^{*}=(V, E)$ such that $V=\{x, y, z, w\}, E=\{(x, y),(y, z),(x, z),(z, w)\}$. Let $A$ be a bipolar fuzzy set of $V$ and let $B$ be a bipolar fuzzy set of $E \subseteq V \times V$ defined by

$$
\begin{gathered}
\mu_{A}^{P}(x)=\mu_{A}^{P}(y)=\mu_{A}^{P}(z)=\mu_{A}^{P}(w)=1, \mu_{A}^{N}(x)=\mu_{A}^{N}(y)=\mu_{A}^{N}(z)=\mu_{A}^{N}(w)=-1 \\
\mu_{B}^{P}(x, y)=\mu_{B}^{P}(y, z)=0.1, \mu_{B}^{P}(x, z)=\mu_{B}^{P}(w, z)=0.9, \mu_{B}^{N}(x, y)=\mu_{B}^{N}(y, z)=-0.5, \mu_{B}^{N}(x, z)=\mu_{B}^{N}(w, z)=-0.1
\end{gathered}
$$

By routine computations, we have $d(B)=-0.5, h(B)=0.9$. For $0<s \leq 0.1, \quad-0.5 \leq t<0$, $G^{(s, t)}=(V,\{(x, y),(y, z),(x, z),(z, w)\})$. For $0.1<s \leq 0.9,-0.1 \leq t<0, G^{(s, t)}=(V,\{(x, z),(z, w)\})$. Thus $(z, w)$ is a full bipolar fuzzy bridge and $(x, z)$ is a partial bipolar fuzzy bridge, but not a full bipolar fuzzy bridge.

We state the following propositions without their proofs.

Proposition 1. Let $(x, y)$ be a bridge in $G^{*}$. Then $(x, y)$ is a bipolar fuzzy bridge if and only if $\mu_{B}^{P}(x, y)>\mu^{\prime}{ }_{B}^{\infty}(x, y)$ and $\mu_{B}^{N}(x, y)<\mu^{N}{ }_{B}^{\infty}(x, y)$.

Proposition 2. $(x, y)$ is a bipolar fuzzy bridge if and only if $(x, y)$ is not a weakest bridge of any cycle.

Proposition 3. $(x, y)$ is a bipolar fuzzy bridge if and only if $(x, y)$ is a bridge for $G^{*}$ and $\mu_{B}^{P}(x, y)=h(B), \mu_{B}^{N}(x, y)=d(B)$.

Proof. Suppose $(x, y)$ is a full bridge. Then $(x, y)$ is a bridge for $G^{(s, t)} \forall(s, t) \in(0, h(B)] \times(0, d(B)]$. Hence $(x, y) \in B^{h(B)}$ and so $\mu_{B}^{P}(x, y)=h(B), \mu_{B}^{N}(x, y)=d(B)$. Since $(x, y)$ is a bridge for $G^{(s, t)}$ for all $(s, t) \in(0, h(B)] \times(0, d(B)]$, it follows that $(x, y)$ is a bridge for $G^{*}$ since $V=A^{d(B)}$ and $E=B^{h(B)}$.

Conversely, suppose that $(x, y)$ is a bridge for $G^{*}$ and $\mu_{B}^{P}(x, y)=h(B), \quad \mu_{B}^{N}(x, y)=d(B)$. Then $(x, y) \in B^{(s, t)} \forall(s, t) \in(0, h(B)] \times[d(B), 0)$. Thus since also $(x, y)$ is a bridge for $G^{*},(x, y)$ is a bridge for $G^{(s, t)} \forall(s, t) \in(0, h(B)] \times[d(B), 0)$ since each $G^{(s, t)}$ is a subgraph of $G^{*}$. Hence $(x, y)$ is a full bipolar fuzzy bridge.

Proposition 4. Suppose that $(x, y)$ is not contained in a cycle of $G^{*}$. Then the following conditions are equivalent:

(1) $\mu_{B}^{P}(x, y)=h(B), \mu_{B}^{N}(x, y)=d(B)$.

(2) $(x, y)$ is a partial bipolar fuzzy bridge.

(3) $(x, y)$ is a bipolar full fuzzy bridge.

Proof. Since $(x, y)$ is not contained in a cycle of $G^{*},(x, y)$ is a bridge of $G^{*}$. Hence by Proposition $3.9,(1) \Leftrightarrow(3)$. Clearly, (3) $\Leftrightarrow$ (2). Suppose that (2) holds. Then $(x, y)$ is a bridge for $G^{(s, t)} \forall(s, t) \in(d(B), h(B)]$ and so $(x, y) \in B^{h(B)}$. Thus $\mu_{B}^{P}(x, y)=h(B), \mu_{B}^{N}(x, y)=d(B)$, i.e., (1) holds.

Proposition 5. If $(x, y)$ is a bridge, then $(x, y)$ is a weak bipolar fuzzy bridge and a bipolar fuzzy bridge.

Proposition 6. $(x, y)$ is a bipolar fuzzy bridge if and only if $(x, y)$ is a weak bridge.

Proof. Suppose $(x, y)$ is a weak bipolar fuzzy bridge. Then $\exists(s, t) \in(0, h(B)] \times[d(B), 0)$ such that $(x, y)$ is a bridge for $G^{(s, t)}$. Hence removal of $(x, y)$ disconnects $G^{(s, t)}$. Thus any path from $x$ to $y$ in $G$ has an edge $(u, v)$ with $\mu_{B}^{P}(u, v)<s$, $\mu_{B}^{N}(u, v)>t$. Thus the removal of $(x, y)$ results in $\left(\mu_{B}^{P}\right)^{\prime \infty}(x, y)<s \leqslant\left(\mu^{P}\right)^{\infty}(x, y),\left(\mu_{B}^{N}\right)^{\prime \infty}(x, y)<t \leqslant\left(\mu^{N}\right)^{\infty}(x, y)$. Hence $(x, y)$ is a bipolar fuzzy bridge. 
Conversely, suppose $(x, y)$ is a bipolar fuzzy bridge. Then $\exists(u, v)$ such that removal of $(x, y)$ results in $\left(\mu_{B}^{P}\right)^{\prime \infty}(u, v)<\left(\mu_{B}^{P}\right)^{\infty}(u, v),\left(\mu_{B}^{N}\right)^{\prime \infty}(u, v)>\left(\mu_{B}^{N}\right)^{\infty}(u, v)$. Hence $(x, y)$ is on every strongest path connecting $u$ and $v$ and in fact, $\mu_{B}^{P}(u, v) \geqslant$ and $\mu_{B}^{N}(u, v) \leqslant$ this value. Thus there does not exist a path (other than $(x, y)$ ) connecting $x$ and $y$ in $G^{\left(\mu_{B}^{P}(x, y), \mu_{B}^{N}(x, y)\right)}$, else this other path without $(x, y)$ would be of strength $\geqslant \mu_{B}^{P}(x, y), \leqslant \mu_{B}^{N}(x, y)$ and would be part of a path connecting $u$ and $v$ of strongest length, contrary to the fact $(x, y)$ is on every such path. Hence $(x, y)$ is a bridge of $G^{\left(\mu_{B}^{P}(x, y), \mu_{B}^{N}(x, y)\right)}$ and $0<\mu_{B}^{P}(x, y) \leq h(B), 0<\mu_{B}^{N}(x, y) \leq d(B)$. Thus $\mu_{B}^{P}(x, y)$ and $\mu_{B}^{N}(x, y)$ are desired $(s, t)$.

Definition 14.[3] A vertex $x \in V$ in $G$ is called $\mu^{P}$-cut vertex, if deleting it reduce the $\mu^{P}$-strength of connectedness between some pair of vertices. A vertex $x \in V$ in called $\mu^{N}$-cut vertex, if deleting it increase the $\mu^{N}$-strength of connectedness between some pair of vertices. A vertex $x \in V$ is a bipolar fuzzy cut vertex, if it is $\mu^{P}$-cut vertex and $\mu^{N}$-cut vertex.

Definition 15. Let $x \in V$.

(1) $x$ is called a cut-vertex if $x$ is a cut-vertex of $G^{*}=\left(A^{*}, B^{*}\right)$.

(2) $x$ is called an bipolar fuzzy cut-vertex if $\left(\mu^{\prime}{ }_{B}\right)^{\infty}(u, v)<\left(\mu_{B}^{P}\right)^{\infty}(u, v)$ and $\left(\mu^{\prime}{ }_{B}\right)^{\infty}(u, v)>\left(\mu_{B}^{N}\right)^{\infty}(u, v)$ for some $u, v \in V$, where $\mu^{P}{ }_{B}$ and $\mu^{N}{ }_{B}$ are $\mu_{B}^{P}$ and $\mu_{B}^{N}$ restricted to $V \times V-\{(x, z),(z, x) \mid z \in V\}$.

(3) $x$ is called a weak bipolar fuzzy cut-vertex if there exists $(s, t) \in(0, h(B)] \times[d(B), 0)$ such that $x$ is a cut-vertex of $G^{(s, t)}$.

(4) $x$ is called a partial bipolar fuzzy cut-vertex if $x$ is a cut-vertex for $G^{(s, t)}$ for all $(s, t) \in(d(B), h(B)] \cup\{h(B)\}$.

(5) $x$ is called a full bipolar fuzzy cut-vertex if $x$ is a cut-vertex for $G^{(s, t)}$ for all $(s, t) \in(0, h(B)] \times[d(B), 0)$.

Example 5. Consider a connected bipolar fuzzy graph as shown in Fig. 2.

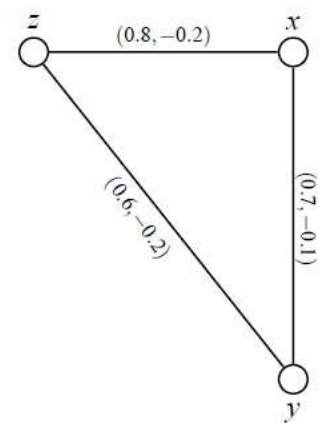

By routine computations, we have $d(B)=-0.2, h(B)=0.8$. Thus $(s, t) \in(0,0.8] \times[-0.1,0)$. For $0<s \leq 0.6,-0.2 \leq t<0$, $G^{(s, t)}=(V,\{(x, y),(y, z),(x, z)\})$. For $0.6<s \leq 0.7,-0.2 \leq t<0, G^{(s, t)}=(V,\{(x, y),(x, z)\})$. For $0.6<s \leq 0.8,-0.1 \leq t<$ $0, G^{(s, t)}=(V,\{(x, z)\})$. Thus $x$ is a bipolar fuzzy cut-vertex and a weak bipolar fuzzy cut-vertex, but neither a cut-vertex nor a partial cut-vertex.

Example 6. Consider a connected graph $G^{*}=(V, E)$ such that $V=\{x, y, z\}, E=\{(x, y),(y, z),(x, z)\}$. Let $A$ be a bipolar fuzzy set of $V$ and let $B$ be a bipolar fuzzy set of $E \subseteq V \times V$ defined by

$$
\begin{gathered}
\mu_{A}^{P}(x)=\mu_{A}^{P}(y)=\mu_{A}^{P}(z)=1, \mu_{A}^{N}(x)=\mu_{A}^{N}(y)=\mu_{A}^{N}(z)=-1, \\
\mu_{B}^{P}(x, y)=\mu_{B}^{P}(x, z)=0.9, \mu_{B}^{P}(y, z)=0.5 \mu_{B}^{N}(x, y)=\mu_{B}^{N}(x, z)=-0.1, \mu_{B}^{N}(y, z)=-0.4 .
\end{gathered}
$$


By routine computations, we have $d(B)=-0.4, h(B)=0.9$. For $0<s \leq 0.5,-0.4 \leq t<0$, $G^{(s, t)}=(V,\{(x, y),(y, z),(x, z)\})$. For $0.5<s \leq 0.9,-0.1 \leq t<0, G^{(s, t)}=(V,\{(x, y),(x, z)\})$. Thus $x$ is a bipolar fuzzy cut-vertex and a partial bipolar fuzzy cut-vertex, but neither a cut-vertex nor a full cut-vertex.

Example 7. Consider a connected graph $G^{*}=(V, E)$ such that $V=\{x, y, z\}, E=\{(x, y),(y, z),(x, z)\}$. Let $A$ be a bipolar fuzzy set of $V$ and let $B$ be a bipolar fuzzy set of $E \subseteq V \times V$ defined by

$$
\begin{gathered}
\mu_{A}^{P}(x)=\mu_{A}^{P}(y)=\mu_{A}^{P}(z)=1, \mu_{A}^{N}(x)=\mu_{A}^{N}(y)=\mu_{A}^{N}(z)=-1, \\
\mu_{B}^{P}(x, y)=\mu_{B}^{P}(x, z)=0.9, \quad \mu_{B}^{N}(x, y)=\mu_{B}^{N}(x, z)=-0.1 .
\end{gathered}
$$

By routine computations, we have $d(B)=-0.1, h(B)=0.9$. For $0<s \leq 0.9,-0.1 \leq t<0, G^{(s, t)}=(V,\{(x, y),(x, z)\})$. Thus $x$ is a full bipolar fuzzy cut-vertex, a bipolar fuzzy cut-vertex and a cut-vertex.

We state the following propositions without their proofs.

Proposition 7. Let $G$ be a bipolar fuzzy graph such that $G^{*}$ is a cycle. Then a node is a bipolar fuzzy cut-node of $G$ if and only if it is a common node of two bipolar fuzzy bridges.

Proposition 8. If $z$ is a common node of at least two bipolar fuzzy bridges, then $z$ is a bipolar fuzzy cut-node.

Proposition 9. If $G$ is a complete bipolar fuzzy graph, then $\left(\mu_{B}^{P}\right)^{\infty}(u, v)=\mu_{B}^{P}(u, v)$ and $\left(\mu_{B}^{N}\right)^{\infty}(u, v)=\mu_{B}^{N}(u, v)$.

Proposition 10. A complete bipolar fuzzy graph has no bipolar fuzzy cut-vertex.

\section{Definition 16.}

(1) $G$ is called a block if $G^{*}$ is a block.

(2) $G$ is called an bipolar fuzzy block if it has no bipolar fuzzy cut-vertices.

(3) $G$ is called a weak bipolar fuzzy block if there exists $(s, t) \in(0, h(B)] \times[d(B), 0)$ such that $G^{(s, t)}$ is a block.

(4) $G$ is called a partial bipolar fuzzy block if $G^{(s, t)}$ is a block for for all $(s, t) \in(d(B), h(B)] \cup\{h(B)\}$.

(5) $G$ is called a full bipolar fuzzy block if $G^{(s, t)}$ is a block for all $(s, t) \in(0, h(B)] \times[d(B), 0)$.

Example 8. Consider a connected bipolar fuzzy graph as shown in the Fig. 3.

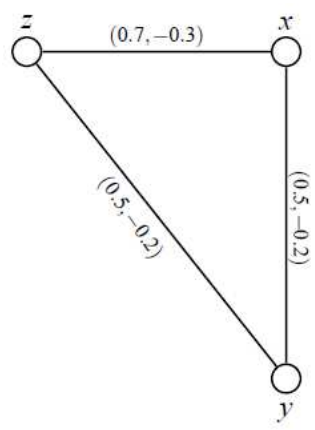

Fig. 3: Connected bipolar fuzzy graph.

By routine computations, we have $d(B)=-0.3, h(B)=0.7$. Thus $(s, t) \in(0,0.7] \times[-0.2,0)$. For $0<s \leq 0.5,-0.3 \leq$ $t<0, G^{(s, t)}=(V,\{(x, y),(y, z),(x, z)\})$. For $0.5<s \leq 0.7,-0.2 \leq t<0, G^{(s, t)}=(V,\{(x, z)\})$. Thus $G$ is a block, a 
bipolar fuzzy block and a weak bipolar fuzzy block. $G$ is not a partial bipolar fuzzy block since $G^{(s, t)}$ is not a block for $0.5<s \leq 0.7,-0.2 \leq t<0$.

Example 9. Consider a connected graph $G^{*}=(V, E)$ such that $V=\{x, y, z\}, E=\{(x, y),(y, z),(x, z)\}$. Let $A$ be a bipolar fuzzy set of $V$ and let $B$ be a bipolar fuzzy set of $E \subseteq V \times V$ defined by

$$
\begin{gathered}
\mu_{A}^{P}(x)=\mu_{A}^{P}(y)=\mu_{A}^{P}(z)=1, \mu_{A}^{N}(x)=\mu_{A}^{N}(y)=\mu_{A}^{N}(z)=-1 \\
\mu_{B}^{P}(x, y)=\mu_{B}^{P}(x, z)=0.9, \mu_{B}^{P}(y, z)=0.5 \mu_{B}^{N}(x, y)=\mu_{B}^{N}(x, z)=-0.1, \mu_{B}^{N}(y, z)=-0.4 .
\end{gathered}
$$

By routine computations, we have $d(B)=-0.4, h(B)=0.9$. For $0<s \leq 0.5,-0.4 \leq t<00$, $G^{(s, t)}=(V,\{(x, y),(y, z),(x, z)\})$. For $0.5<s \leq 0.9,-0.1 \leq t<0, G^{(s, t)}=(V,\{(x, y),(x, z)\})$. Thus $G$ is a block and a weak bipolar fuzzy block. However, $G$ is not a bipolar fuzzy block since $x$ is a bipolar fuzzy cut-vertex of $G$. Also $G$ is not a partial bipolar fuzzy block since $x$ is a cut-vertex for $0.5<s \leq 0.9,-0.1 \leq t<0$.

Example 10. Consider a connected graph $G^{*}=(V, E)$ such that $V=\{x, y, z\}, E=\{(x, y),(y, z),(x, z)\}$. Let $A$ be a bipolar fuzzy set of $V$ and let $B$ be a bipolar fuzzy set of $E \subseteq V \times V$ defined by

$$
\begin{gathered}
\mu_{A}^{P}(x)=\mu_{A}^{P}(y)=\mu_{A}^{P}(z)=1, \mu_{A}^{N}(x)=\mu_{A}^{N}(y)=\mu_{A}^{N}(z)=-1 \\
\mu_{B}^{P}(x, y)=\mu_{B}^{P}(x, z)=\mu_{B}^{P}(y, z)=0.9 \mu_{B}^{N}(x, y)=\mu_{B}^{N}(x, z)=\mu_{B}^{N}(y, z)=-0.1 .
\end{gathered}
$$

By routine computations, we have $d(B)=-0.1, h(B)=0.9$. For $0<s \leq 0.9,-0.1 \leq t<0$, $G^{(s, t)}=(V,\{(x, y),(y, z),(x, z)\})$. Thus $G$ is a block, a bipolar fuzzy block and a full bipolar fuzzy block.

Definition 17. A connected bipolar fuzzy graph $G$ is said to be firm if

$$
\begin{aligned}
& \min \left\{\mu_{A}^{P}(x) \mid x \in V\right\} \geq \max \left\{\mu_{B}^{P}(x, y) \mid(x, y) \in E\right\}, \\
& \max \left\{\mu_{A}^{N}(x) \mid x \in V\right\} \leq \min \left\{\mu_{B}^{N}(x, y) \mid(x, y) \in E\right\} .
\end{aligned}
$$

Example 11. All connected bipolar fuzzy graphs as shown in the Fig. 1, Fig. 2 and Fig. 3 are firms.

Example 12. Consider a connected bipolar fuzzy graph as shown in the Fig. 4.

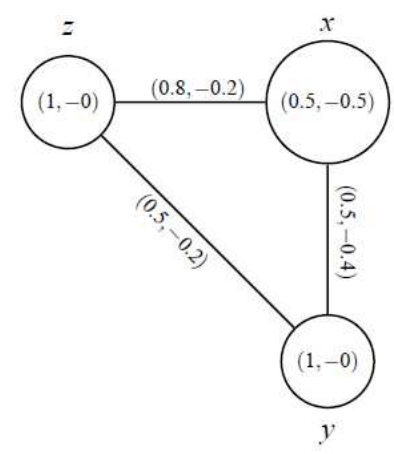

Fig. 4: Connected bipolar fuzzy graph. 
By routine computations, we have $d(B)=-0.4, h(B)=0.8$. Thus $(s, t) \in(0,-0.8] \times(0,-0.2]$. For $0<s \leq 0.5,-0.4 \leq$ $t<0, G^{(s, t)}=(V,\{(x, y),(y, z),(x, z)\})$. For $0.5<s \leq 0.8,-0.2 \leq t<0, G^{(s, t)}=(V,\{(x, z)\})$. Thus $G$ is a block, a bipolar fuzzy block and full bipolar fuzzy block. We note that $G$ is not firm.

\section{Cycles and trees}

\section{Definition 18.}

(1) $G$ is called a cycle if $G^{*}$ is a cycle.

(2) $G$ is called an bipolar fuzzy cycle if $G^{*}$ is a cycle and there does not exist unique $(x, y) \in E$ such that $\mu_{B}^{P}(x, y)=$ $\min \left\{\mu_{B}^{P}(u, v) \mid(u, v) \in E\right\}, \mu_{B}^{N}(x, y)=\max \left\{\mu_{B}^{N}(u, v) \mid(u, v) \in E\right\}$.

(3) $G$ is called a weak bipolar fuzzy cycle if there exists $(s, t) \in(0, h(B)] \times[d(B), 0)$ such that $G^{(s, t)}$ is a cycle.

(4) $G$ is called a partial bipolar fuzzy cycle if $G^{(s, t)}$ is a cycle for for all $(s, t) \in(d(B), h(B)] \cup\{h(B)\}$.

(5) $G$ is called a full bipolar fuzzy cycle if $G^{(s, t)}$ is a cycle for all $(s, t) \in(0, h(B)] \times[d(B), 0)$.

Example 13. Consider a connected bipolar fuzzy graph as shown in Fig. 5.

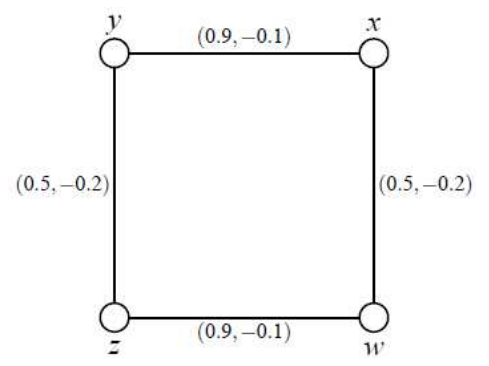

Fig. 5: Connected bipolar fuzzy graph.

By routine computations, we have $d(B)=-0.2, h(B)=0.9$. Thus $(s, t) \in(0,-0.9] \times(0,-0.1]$. For $0<s \leq 0.5,0 \leq t<$ $-0.2, G^{(s, t)}=(V,\{(x, y),(x, w),(y, z),(w, z)\})$. For $0.5<s \leq 0.9,-0.1 \leq t<0, G^{(s, t)}=(V,\{(x, y),(z, w)\})$. Thus $G$ is a bipolar fuzzy cycle and weak bipolar fuzzy cycle but $G$ is not partial bipolar fuzzy cycle.

Example 14. Consider a connected bipolar fuzzy graph as shown in the Fig. 6.

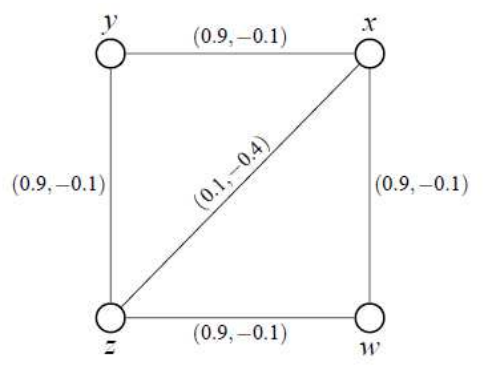

Fig. 6: Connected bipolar fuzzy graph. 
By routine computations, we have $d(B)=-0.4, h(B)=0.9$. Thus $(s, t) \in(0, h(B)] \times[d(B), 0)$ means $(s, t) \in(0,-0.9] \times$ $[-0.1,0)$. For $0<s \leq 0.1,-0.4 \leq t<0, G^{(s, t)}=(V,\{(x, y),(y, z),(w, z),(w, x),(x, w)\})$ which is not a cycle. For $0.1<$ $s \leq 0.9,-0.1 \leq t<0, G^{(s, t)}=(V,\{(x, y),(y, z),(z, w),(w, x)\})$ which is a cycle. Thus $G$ not cycle, $G$ is a partial bipolar fuzzy cycle but not a full bipolar fuzzy cycle.

The proofs of the following propositions are trivial.

Proposition 11. Suppose $G$ is a cycle. Then $G$ is a partial bipolar fuzzy cycle if and only if $G$ is a full bipolar fuzzy cycle.

Proposition 12. $G$ is a full bipolar fuzzy cycle if and only if $G$ is a cycle and $B$ is constant on $E$.

Definition 19. A connected bipolar fuzzy graph $G=(A, B)$ is a bipolar fuzzy tree if it has a bipolar fuzzy spanning subgraph $H=(A, C)$ which is a tree, where for all arcs $(x, y)$ not in $H, \mu_{B}^{P}(x, y)<\left(\mu_{B}^{P}\right)_{C}^{\infty}(x, y), \mu_{B}^{N}(x, y)>\left(\mu_{B}^{N}\right)_{C}^{\infty}(x, y)$.

\section{Definition 20.}

(1) $G$ is called a forest if $G^{*}$ is a forest.

(2) $G$ is called a bipolar fuzzy forest if $G$ has a bipolar fuzzy spanning subgraph $H=(A, C)$ which is a forest such that for all $(u, v) \in E-W, \mu_{B}^{P}(u, v)<\left(\mu_{C}^{P}\right)^{\infty}(u, v)$ and $\mu_{B}^{N}(u, v)>\left(\mu_{C}^{N}\right)^{\infty}(u, v)$.

(3) $G$ is called a weak bipolar fuzzy forest if for all $(s, t) \in(0, h(B)] \times[d(B), 0)$ such that $G^{(s, t)}$ is a forest.

(4) $G$ is called a partial bipolar fuzzy forest if $G^{(s, t)}$ is a forest for for all $(s, t) \in(d(B), h(B)] \cup\{h(B)\}$.

(5) $G$ is called a full bipolar fuzzy forest if $G^{(s, t)}$ is a forest for all $(s, t) \in(0, h(B)] \times[d(B), 0)$.

Example 15. Consider a connected graph $G^{*}=(V, E)$ such that $V=\{x, y, z, w\}, E=\{(x, y),(y, z),(x, w),(w, z)\}$. Let $A$ be a bipolar fuzzy set of $V$ and let $B$ be a bipolar fuzzy set of $E \subseteq V \times V$ defined by

$$
\begin{gathered}
\mu_{A}^{P}(x)=\mu_{A}^{P}(y)=\mu_{A}^{P}(z)=\mu_{A}^{P}(w)=1, \mu_{A}^{N}(x)=\mu_{A}^{N}(y)=\mu_{A}^{N}(z)=\mu_{A}^{N}(w)=-1, \\
\mu_{B}^{P}(x, y)=\mu_{B}^{P}(w, z)=0.9, \mu_{B}^{P}(x, w)=\mu_{B}^{P}(y, z)=0.5, \mu_{B}^{N}(x, y)=\mu_{B}^{N}(w, z)=-0.1, \mu_{B}^{N}(x, w)=\mu_{B}^{N}(y, z)=-0.4 .
\end{gathered}
$$

By routine computations, we have $d(B)=-0.4, h(B)=0.9$. For $0<s \leq 0.5, \quad-0.4 \leq t<0$, $G^{(s, t)}=(V,\{(x, w),(y, z),(x, y),(w, z)\})$, and for $0.5<s \leq 0.9,-0.1 \leq t<0, G^{(s, t)}=(V,\{(x, y),(w, z)\})$. Thus $G$ is a partial bipolar fuzzy forest, but is neither a bipolar fuzzy forest nor a full bipolar fuzzy forest.

Proposition 13. $G$ is a full bipolar fuzzy forest if and only if $G$ is forest.

Proof. Suppose that $G$ is a full bipolar fuzzy forest. Then $G^{*}=G^{d(B)}$ is a forest.

Conversely, suppose that $G$ is a forest. Then $G^{*}$ is a forest and hence so must be $G^{(s, t)}$ for all $(s, t) \in(0, h(B)] \times[d(B), 0)$ since each such $G^{(s, t)}$ is a subgraph of $G^{*}$. This completes the proof.

Example 16. Consider a connected graph $G^{*}=(V, E)$ such that $V=\{x, y, z\}, E=\{(x, y),(y, z),(x, z)\}$. Let $A$ be a bipolar fuzzy set of $V$ and let $B$ be a bipolar fuzzy set of $E \subseteq V \times V$ defined by

$$
\begin{gathered}
\mu_{A}^{P}(x)=\mu_{A}^{P}(y)=\mu_{A}^{P}(z)=1, \mu_{A}^{N}(x)=\mu_{A}^{N}(y)=\mu_{A}^{N}(z)=-1, \\
\mu_{B}^{P}(x, y)=0.9, \mu_{B}^{P}(y, z)=0.5, \mu_{B}^{N}(x, y)=-0.1, \mu_{B}^{N}(y, z)=-0.4 .
\end{gathered}
$$

By routine computations, we have $d(B)=(0.5,-0.4), h(B)=(0.9,-0.1)$. For $0<s \leq 0.5,-0.4 \leq t<0, G^{(s, t)}=$ $(V,\{(x, y),(y, z)\})$. For $0.5<s \leq 0.9,-0.1 \leq t<0, G^{(s, t)}=(V,\{(x, y)\})$. Thus $G$ is a forest and a full bipolar fuzzy forest without being a constant on $E$. Note that $G^{h}(B)$ has more connected components than $G^{*}$. 
Proposition 14. $G$ is a weak bipolar fuzzy forest if and only if $G$ does not contain a cycle whose edges are of strength $h(B)$.

Proof. Suppose $G$ contains a cycle whose edges are of strength $h(B)$. Then $G^{(s, t)},(s, t) \in(0, h(B)]$ contains this cycle and so is not a forest. Thus $G$ is not a weak bipolar fuzzy forest.

Conversely, suppose $G$ does not contain a cycle all of whose edges are of strength $h(B)$. Then $G^{h(B)}$ does not contain a cycle and so is a forest.

Corollary 1. If $G$ is a bipolar fuzzy forest, then $G$ is a weak bipolar fuzzy forest.

Theorem 1. $G$ is a forest and $B$ is constant on $E$ if and only if $G$ is a full bipolar fuzzy forest, $G^{*}$ and $G^{h(B)}$ have the same number of connected components and $G$ is firm.

Proof. Suppose that $G$ is a forest and $B$ is constant on $E$. Then $\forall(s, t) \in(0, h(B)], G^{(s, t)}=G^{*}$ and so $G$ is a full bipolar fuzzy forest and $G^{*}$ and $G^{h(B)}$ have the same number of connected components. Clearly, $G$ is firm since $B$ is a constant on $E$.

Conversely, suppose $G$ is a full bipolar fuzzy forest, $G^{*}$ and $G^{h(B)}$ have the same number of connected components, and $G$ is firm. Suppose $\exists\left(s_{1}, t_{1}\right),\left(s_{2}, t_{2}\right) \in \operatorname{lm}(B)$ such that $0<s_{1}, s_{2}, t_{1}, t_{2}$. Then $\exists(x, y) \in E$ such that $\mu_{B}^{P}(x, y)=S_{1}$, $\mu_{B}^{N}(x, y)=t_{1}$. Now $(x, y) \in B^{\left(s_{1}, t_{1}\right)},(x, y) \notin B^{\left(s_{2}, t_{2}\right)}$. Hence $G^{\left(s_{2}, t_{2}\right)}$ has more connected components then $G^{\left(s_{1}, t_{1}\right)}$ since $G$ is firm, i.e., no vertices were lost. Thus $G^{h(B)}$ has more connected components than $G^{*}$, a contradiction.

Corollary 2. $G$ is a tree and $B$ is constant on $E$ if and only if $G$ is a full bipolar fuzzy tree and $G$ is firm.

\section{Definition 21.}

(1) $G$ is called a tree if $G^{*}$ is a tree.

(2) $G$ is called a bipolar fuzzy tree if $G$ has a bipolar fuzzy spanning subgraph $H=(A, C)$ which is a tree such that for all $(u, v) \in E-W, \mu_{B}^{P}(u, v)<\left(\mu_{C}^{P}\right)^{\infty}(u, v)$ and $\mu_{B}^{N}(u, v)>\left(\mu_{C}^{N}\right)^{\infty}(u, v)$.

(3) $G$ is called a weak bipolar fuzzy tree if for all $(s, t) \in(0, h(B)] \times[d(B), 0)$ such that $G^{(s, t)}$ is a tree.

(4) $G$ is called a partial bipolar fuzzy tree if $G^{(s, t)}$ is a tree for for all $(s, t) \in(d(B), h(B)] \cup\{h(B)\}$.

(5) $G$ is called a full bipolar fuzzy tree if $G^{(s, t)}$ is a tree for all $(s, t) \in(0, h(B)] \times[d(B), 0)$.

Example 17. Consider a connected graph $G^{*}=(V, E)$ such that $V=\{x, y, z\}, E=\{(x, y),(y, z),(x, z)\}$. Let $A$ be a bipolar fuzzy set of $V$ and let $B$ be a bipolar fuzzy set of $E \subseteq V \times V$ defined by

$$
\begin{gathered}
\mu_{A}^{P}(x)=\mu_{A}^{P}(y)=1, \mu_{A}^{P}(z)=0.5, \mu_{A}^{N}(x)=\mu_{A}^{N}(y)=0, \mu_{A}^{N}(z)=-0.2, \\
\mu_{B}^{P}(x, y)=0.9, \mu_{B}^{P}(y, z)=0.5, \mu_{B}^{N}(x, y)=0.1, \mu_{B}^{N}(y, z)=-0.4 .
\end{gathered}
$$

By routine computations, we have $d(B)=-0.4, h(B)=0.9$. For $0<s \leq 0.9,-0.1 \leq t<0, G^{(s, t)}=(V,\{(x, y),(y, z)\})$, and for $0.5<s \leq 0.9,-0.1 \leq t<0, G^{(s, t)}=(V,\{(x, y)\})$. Thus $G$ is a tree, $G$ is a full bipolar fuzzy tree, and $G^{*}$ and $G^{h}(B)$ has the same number of connected components. However, $G$ is not firm and $B=\left(\mu_{B}^{P}, \mu_{B}^{N}\right)$ is not constant on $E$.

Example 18. Consider a connected graph $G^{*}=(V, E)$ such that $V=\{x, y, z\}, E=\{(x, y),(y, z),(x, z)\}$. Let $A$ be a bipolar fuzzy set of $V$ and let $B$ be a bipolar fuzzy set of $E \subseteq V \times V$ defined by

$$
\begin{gathered}
\mu_{A}^{P}(x)=\mu_{A}^{P}(y)=1, \mu_{A}^{P}(z)=0.5, \mu_{A}^{N}(x)=\mu_{A}^{N}(y)=-1, \mu_{A}^{N}(z)=-0.2, \\
\mu_{B}^{P}(x, y)=0.9, \mu_{B}^{P}(x, z)=\mu_{B}^{P}(y, z)=0.5, \mu_{B}^{N}(x, y)=-0.1, \mu_{B}^{N}(x, z)=\mu_{B}^{N}(y, z)=-0.4 .
\end{gathered}
$$


By routine computations, we have $d(B)=-0.4, h(B)=0.9$. For $0<s \leq 0.5,-0.4 \leq t<0$, $G^{(s, t)}=(V,\{(x, y),(x, z),(y, z)\})$, and for $0.5<s \leq 0.9,-0.1 \leq t<0, G^{(s, t)}=(\{x, y\},\{(x, y)\})$. Thus $G$ is a partial bipolar fuzzy tree, but not a full bipolar fuzzy tree. $G$ is not a bipolar fuzzy tree.

We state the following propositions without their proofs.

Proposition 15. If $G$ is a bipolar fuzzy tree, then $G$ is not complete.

Proposition 16. If $G$ is a bipolar fuzzy tree, then arcs of spanning subgraph $H$ are the bipolar fuzzy bridges of $G$.

Proposition 17. If $G$ is a bipolar fuzzy tree, then internal nodes of spanning subgraph $H$ are the bipolar fuzzy cutnodes of G.

Proposition 18. $G$ is a bipolar fuzzy tree if and only if the following are equivalent:

(a) $(x, y)$ is a bipolar fuzzy bridge.

(b) $\left(\mu_{B}^{P}\right)^{\infty}(x, y)=\mu_{B}^{P}(x, y)$ and $\left(\mu_{B}^{N}\right)^{\infty}(x, y)=\mu_{B}^{N}(x, y)$.

Proposition 19. A bipolar fuzzy graph is a bipolar fuzzy tree if and only if it has a unique maximum spanning tree.

Proposition 20. Suppose that $G$ is firm. If $G$ is a weak bipolar fuzzy tree, then $G$ is a bipolar fuzzy tree.

Proof. There exist $(s, t) \in(\mathbf{0}, h(B)]$ such that $G^{(s, t)}$ is a tree. Since $G$ is firm, $G^{(s, t)}$ is a bipolar fuzzy spanning subgraph of $G$ which is a tree. If $(u, v)$ is in $E B^{(s, t)}$, then $\mu_{B}^{P}(u, v)<s, \mu_{B}^{N}(u, v)>t$ and so it follows that $G$ is a bipolar fuzzy tree.

\section{Definition 22.}

(1) $G$ is called a connected if $G^{*}$ is a connected.

(2) $G$ is called a bipolar fuzzy connected if $G$ is a bipolar fuzzy block.

(3) $G$ is called a weak bipolar fuzzy connected if there exists $(s, t) \in(0, h(B)] \times[d(B), 0)$ such that $G^{(s, t)}$ is a connected.

(4) $G$ is called a partial bipolar fuzzy connected if $G^{(s, t)}$ is a connected for for all $(s, t) \in(d(B), h(B)] \cup\{h(B)\}$.

(5) $G$ is called a full bipolar fuzzy connected if $G^{(s, t)}$ is a connected for all $(s, t) \in(0, h(B)] \times[d(B), 0)$.

Proposition 21. If $G$ is connected, then $G$ is weakly connected.

Proof. $G$ connected implies $G^{*}$ is connected. Now $G^{*}=G^{h(B)}$ and so $G$ is weakly connected.

Proposition 22. If $G$ is firm and weakly connected, then $G$ is connected.

Proof. If $G^{(s, t)}$ is connected for some $(s, t) \in(0, h(B)]$, then $G^{*}$ is connected since $G$ is firm.

\section{Proposition 23.}

(1) If $G$ is a weak bipolar fuzzy tree, then $G$ is weakly connected and $G$ is a weak bipolar fuzzy forest. Conversely, if $\exists\left(s_{1}, t_{1}\right),\left(s_{2}, t_{2}\right) \in(0, h(B)]$ with $s_{1}<s_{2}, t_{1}<t_{2}$ such that $G^{\left(s_{1}, t_{1}\right)}$ is a forest and $G^{\left(s_{2}, t_{2}\right)}$ is connected, then $G$ is a weak bipolar fuzzy tree.

(2) $G$ is a tree if and only if $G$ is a forest and $G$ is connected.

(3) $G$ is partial bipolar fuzzy tree if and only if $G$ is a partial bipolar fuzzy forest and $G$ is a partially bipolar fuzzy connected.

(4) $G$ is a full bipolar fuzzy tree if and only if $G$ is a full bipolar fuzzy forest and $G$ is fully connected.

Proof. (1) If $G^{(s, t)}$ is a tree for some $(s, t) \in(\mathbf{0}, h(B)]$, then $G^{(s, t)}$ is connected and is a forest. For the converse, we note that $G^{\left(s_{2}, t_{2}\right)}$ must also be a forest. Since also $G^{\left(s_{2}, t_{2}\right)}$ is connected, $G^{\left(s_{2}, t_{2}\right)}$ is a tree.

The proofs of (2),(3) and(4)are immediate. 
Proposition 24. $G$ is a firm if and only if $G^{(s, t)}$ is firm $\forall(s, t) \in(\mathbf{0}, h(B)]$.

Proof. Suppose $G$ is firm. Let $(s, t) \in(0, h(B)] \times[d(B), 0)$. Let $(x, y) \in\left(\mu^{P}\right)^{(s, t)}$. Then

$$
\begin{gathered}
s \leq \mu_{B}^{P}(x, y) \leq \min \left\{\mu_{A}^{P}(x) \mid x \in V\right\} \leq \min \left\{\mu_{A}^{P}(x) \mid x \in\left(\mu_{A}^{P}\right)^{s}\right\}, \\
t \geq \mu_{B}^{N}(x, y) \geq \max \left\{\mu_{A}^{N}(x) \mid x \in V\right\} \geq \max \left\{\mu_{A}^{N}(x) \mid x \in\left(\mu_{A}^{N}\right)^{t}\right\} .
\end{gathered}
$$

Hence $\max \left\{\mu_{B}^{P}(x, y) \mid(x, y) \in\left(\mu_{B}^{P}\right)^{s}\right\} \leq \min \left\{\mu_{A}^{P}(x) \mid x \in\left(\mu_{A}^{P}\right)^{s}\right\}, \min \left\{\mu_{B}^{N}(x, y) \mid(x, y) \in\left(\mu_{B}^{N}\right)^{t}\right\} \geq \max \left\{\mu_{A}^{N}(x) \mid x \in\left(\mu_{A}^{N}\right)^{t}\right\}$. Thus we conclude that $B^{(s, t) *}=B^{(s, t)}$ and $A^{(s, t) *}=A^{(s, t)}, G^{(s, t)}$ is firm.

Conversely, suppose $G^{(s, t)}$ is firm $\forall(s, t) \in(0, h(B)] \times[d(B), 0)$. Let $\min \left\{\mu_{A}^{P}(x) \mid x \in V\right\}=s_{0}, \max \left\{\mu_{A}^{N}(x) \mid x \in V\right\}=t_{0}$. Then $t_{0}>0$. Now $\max \left\{\mu_{B}^{P}(x, y) \mid(x, y) \in\left(\mu_{B}^{P}\right)^{s_{0}}\right\} \leq s_{0}, \min \left\{\mu_{B}^{N}(x, y) \mid(x, y) \in\left(\mu_{B}^{N}\right)^{t_{0}}\right\} \geq t_{0}$ since $G^{\left(s_{0}, t_{0}\right)}$ is firm and $V=$ $A^{\left(s_{0}, t_{0}\right)}=A^{\left(s_{0}, t_{0}\right) *}$. Let $(x, y) \in E-B^{(s, t) *}$. Then $\mu_{B}^{P}(x, y)<s_{0}, \mu_{B}^{N}(x, y)>t_{0}$. Thus

$$
\begin{aligned}
& \max \left\{\mu_{B}^{P}(x, y) \mid(x, y) \in E\right\} \leq s_{0}=\min \left\{\mu_{A}^{P}(x) \mid x \in V\right\}, \\
& \min \left\{\mu_{B}^{N}(x, y) \mid(x, y) \in E\right\} \geq t_{0}=\max \left\{\mu_{A}^{N}(x) \mid x \in V\right\} .
\end{aligned}
$$

Hence $G$ is firm.

\section{Conclusions}

In a network, each arc is assigned a weight. The weight of a path or a cycle is defined as the minimum weight of its arcs. The maximum of weights of all paths between two nodes is defined as the strength of connectedness between the nodes. In network applications, the reduction in the strength of connectedness is more relevant than the total disconnection of the graph. A graph is totally weighted if both node set and arc set are weighted. Fuzzy graph theory is finding an increasing number of applications in modeling real time systems. Since bipolar fuzzy models give more precision, flexibility and compatibility to the system as compared to the fuzzy models, we have investigated some properties of bipolar fuzzy cycles, bipolar fuzzy trees, bipolar fuzzy bridges, and bipolar fuzzy cut-vertices in bipolar fuzzy graphs in this paper. We plan to extend our research of fuzzification to (1) Bipolar fuzzy soft trees, (2) Soft cycles and soft trees, (3), and Rough cycles and rough trees.

\section{References}

[1] M. Akram, Bipolar fuzzy graphs, Information Sciences 181 (2011) 5548-5564.

[2] M. Akram, Bipolar fuzzy graphs with applications, Knowledge Based Systems, 39(2013) 1-8.

[3] M. Akram and W.A. Dudek, Regular bipolar fuzzy graphs, Neural Computing \& Applications 21(2012)197-205.

[4] M. Akram and M.G. Karunambigai, Metric in bipolar fuzzy graphs, World Applied Sciences Journal 14(2011)1920-1927.

[5] M. Akram, S. Li and K. P. Shum, Antipodal bipolar fuzzy graphs, Italian Journal of Pure and Applied Mathematics, 31(2013)425438.

[6] M. Akram, W.A. Dudek and S. Sarwar, Properties of bipolar fuzzy hypergraphs, Italian Journal of Pure and Applied Mathematics,31(2013)426-458.

[7] P. Bhattacharya, Some remarks on fuzzy graphs, Pattern Recognition Letter 6(1987), 297-302.

[8] K.R. Bhutani and A. Rosenfeld, Strong arcs in fuzzy graphs, Information Sciences 152 (2003)319-322.

[9] A. Kauffman, Introduction a la Theorie des Sous-emsembles Flous, Masson et Cie, Vol.1, 1973.

[10] L.T. Koczy, 1Fuzzy graphs in the evaluation and optimization of networks, Fuzzy sets and systems, 46 (3)(1992) 307-319. 
[11] S. Mathew and M.S. Sunitha, Types of arcs in a fuzzy graph, Information Sciences 179(11)(2009) 1760-1768.

[12] K.-M. Lee, Bipolar-valued fuzzy sets and their basic operations, Proc. Int. Conf., Bangkok, Thailand, (2000) 307-317.

[13] J.N. Mordeson and P.S. Nair, Fuzzy graphs and fuzzy hypergraphs, Physica Verlag, Heidelberg 1998; Second Edition 2001.

[14] A. Nagoorgani and V.T. Chandrasekaran A first look at fuzzy graph theory, Allied Publishers Pvt. Ltd, 2010.

[15] A. Rosenfeld, Fuzzy graphs, Fuzzy Sets and their Applications (L.A. Zadeh, K.S. Fu, M. Shimura, Eds.), Academic Press, New York (1975) 77-95.

[16] M.S. Sunitha and A. Vijayakumar, A characterization of fuzzy trees, Information Sciences 113 (1999) 293-300.

[17] A. Rosenfeld, Fuzzy graphs, Fuzzy Sets and their Applications (L.A. Zadeh, K.S. Fu, M. Shimura, Eds.), Academic Press, New York (1975) 77-95.

[18] M.S. Sunitha and A. Vijayakumar, Complement of a fuzzy graph, Indian J. Pure Appl. Math. 33(2002) 1451-1464.

[19] L.A. Zadeh, Fuzzy sets, Information and Control 8(1965) 338-353.

[20] L.A. Zadeh, Similarity relations and fuzzy orderings, Information Sciences 3(2)(1971) 177-200.

[21] W.-R. Zhang, Bipolar fuzzy sets and relations: a computational framework forcognitive modeling and multiagent decision analysis, Proc. of IEEE Conf. (1994) 305-309.

[22] W.-R. Zhang, Bipolar fuzzy sets, Proc. of FUZZ-IEEE (1998) 835-840. 